in vivo $35: 1141-1145(2021)$

doi:10.21873/invivo.12360

\title{
Single Short Retention Instillation of Pirarubicin Prevents Intravesical Recurrence of Low-risk Non Muscle Invasive Bladder Cancer
}

\author{
SUSUMU KAGEYAMA ${ }^{1}$, KOKI MAEDA ${ }^{1,2}$, SHIGEHISA KUBOTA ${ }^{1}$, TETSUYA YOSHIDA ${ }^{1}$, \\ TAKASHI OSAFUNE ${ }^{3}$, YUTAKA ARAI ${ }^{4}$, HIROKI SOGA ${ }^{5}$, ZENKAI NISHIKAWA ${ }^{6}$, YUJI SAKANO ${ }^{7}$, \\ KEITA TAKIMOTO $^{8}$, CHUL JANG KIM $^{9}$, TOKUHIRO CHANO $^{10}$ and AKIHIRO KAWAUCHI ${ }^{1}$ \\ ${ }^{1}$ Department of Urology, Shiga University of Medical Science, Shiga, Japan; \\ ${ }^{2}$ Department of Urology, Nagahama Red Cross Hospital, Shiga, Japan; \\ ${ }^{3}$ Department of Urology, Uji-Tokushukai Medical Center, Kyoto, Japan; \\ ${ }^{4}$ Department of Urology, Kusatsu General Hospital, Shiga, Japan; \\ ${ }^{5}$ Department of Urology, Toyosato Hospital, Shiga, Japan; \\ ${ }^{6}$ Department of Urology, Hino Memorial Hospital, Shiga, Japan; \\ ${ }^{7}$ Department of Urology, Higashi-Ohmi General Medical Center, Shiga, Japan; \\ ${ }^{8}$ Department of Urology, Saiseikai Shiga Hospital, Shiga, Japan; \\ ${ }^{9}$ Department of Urology, Kohka Public Hospital, Shiga, Japan; \\ ${ }^{10}$ Department of Clinical Laboratory Medicine, Shiga University of Medical Science, Shiga, Japan
}

\begin{abstract}
Background: This study evaluated the efficacy of a single instillation of pirarubicin with a short retention time for preventing intravesical recurrence of low-risk nonmuscle-invasive bladder cancer. Patients and Methods: We analyzed 165 patients with low-risk non-muscle-invasive bladder cancer who underwent transurethral surgery. Single instillation of pirarubicin with 15 -min retention time immediate after surgery was performed in 47 (28\%) patients. The other patients $(118,72 \%)$ were treated without instillation therapy. The primary endpoint was recurrencefree survival. Results: Median overall follow-up was 50 (range=6-134) months. Recurrence-free survival at 1 and 5 years was $91 \%$ and $72 \%$, and $79 \%$ and $54 \%$ in the group treated with pirarubicin, and that treated with surgery alone, respectively $(p=0.031)$. Cox's hazard analysis revealed lack of instillation and larger tumor size $(>10 \mathrm{~mm})$ as significant factors for risk of recurrence. No adverse events regarding
\end{abstract}

This article is freely accessible online.

Correspondence: Susumu Kageyama, MD, Ph.D., Associate Professor, Department of Urology, Shiga University of Medical Science, Seta Tsukinowa-cho, Otsu, Shiga 520-2192, Japan. E-mail: kageyama@belle.shiga-med.ac.jp

Key Words: Immediate postoperative single instillation, non-muscleinvasive bladder cancer, pirarubicin. intravesical chemotherapy were observed. Conclusion: Pirarubicin instillation with 15 -min retention time can prevent intravesical recurrence of low-risk bladder tumors.

Bladder cancer is the $10^{\text {th }}$ most common cancer worldwide, with approximately 550,000 new cases diagnosed in 2018 (1). About $75 \%$ of patients have non-muscle-invasive bladder cancer (NMIBC) at initial diagnosis and usually undergo transurethral resection of bladder tumor (TURBT) (2). However, a high intravesical recurrence rate is the major clinical problem, and adjuvant intravesical chemotherapy and immunotherapy are required in order to reduce recurrence and progression. Low-risk NMIBC is defined in clinical guidelines by the presence of small $(<3 \mathrm{~cm})$, solitary, lowgrade non-invasive papillary carcinoma (Ta tumor); the disease is associated with lower intravesical recurrence and progression rates when compared to intermediate- and highrisk NMIBC (3-5). Immediate postoperative single instillation (IPSI) of chemotherapeutic agents is strongly recommended to prevent intravesical recurrence of low-risk NMIBC (3-6). In spite of its established efficacy in reducing intravesical recurrence, IPSI is infrequently used in clinical practice (7).

Pirarubicin (4'-O-tetrahydropyranyladriamycin), a semisynthetic anthracycline glycoside, is a chemotherapeutic agent that is used for intravesical therapy (8). Its prophylactic effect is similar to that of mitomycin C (MMC) and epirubicin (9). Pirarubicin is taken up rapidly in both cultured tumor cells and bladder tumors in situ $(10,11)$. In 
in vivo $35: 1141-1145(2021)$

Table I. Characteristics of patients treated with transurethral resection of bladder tumor (TURBT) only or with TURBT plus pirarubicin.

\begin{tabular}{|c|c|c|c|c|}
\hline & Overall & Pirarubicin & TURBT alone & \\
\hline Case number & 165 & 47 & 118 & $p$-Value \\
\hline \multicolumn{5}{|l|}{ Age, years } \\
\hline Median (range) & $68(40-95)$ & $68(47-90)$ & $68(40-95)$ & 0.884 \\
\hline \multicolumn{5}{|l|}{ Gender, $\mathrm{n}(\%)$} \\
\hline Male & $134(81.2)$ & $41(87.2)$ & $93(78.8)$ & 0.272 \\
\hline Female & $31(18.7)$ & $6(12.8)$ & $25(21.2)$ & \\
\hline \multicolumn{5}{|l|}{ Tumor size, mm } \\
\hline Median (range) & $10(1-25)$ & $10(2-25)$ & $10(1-25)$ & 0.854 \\
\hline \multicolumn{5}{|c|}{ Voided urine cytology, $\mathrm{n}(\%)^{*}$} \\
\hline Positive & $16(14.7)$ & $7(16.7)$ & $9(13.5)$ & 0.782 \\
\hline Negative & $93(85.3)$ & $35(83.3)$ & $58(86.5)$ & \\
\hline \multicolumn{5}{|c|}{ Grade (WHO 1973), n (\%)** } \\
\hline 1 & $91(55.8)$ & $23(50.0)$ & $68(58.1)$ & 0.384 \\
\hline 2 & $72(44.2)$ & $23(50.0)$ & $49(41.9)$ & \\
\hline \multicolumn{5}{|c|}{ Follow-up for censored patients, months } \\
\hline Median (range) & $50(6-134)$ & $51(9-113)$ & $50(6-134)$ & 0.676 \\
\hline
\end{tabular}

Data not available: $* 56$ and $* * 2$ patients.

Table II. Results of univariate and multivariate analyses for intravesical recurrence of non-muscle-invasive bladder cancer.

\begin{tabular}{|c|c|c|c|c|c|}
\hline & & \multicolumn{2}{|c|}{ Univariate } & \multicolumn{2}{|c|}{ Multivariate } \\
\hline & & $p$-Value & HR & $95 \% \mathrm{CI}$ & $p$-Value \\
\hline Age & $\leq 68 v s .>68$ Years & 0.961 & & & \\
\hline Gender & Male $v s$. female & 0.914 & & & \\
\hline Cytology & Negative $v s$. positive & 0.398 & & & \\
\hline Grade & 1 vs. 2 & 0.650 & & & \\
\hline Tumor size & $\leq 10 v s .>10 \mathrm{~mm}$ & 0.002 & 2.33 & $1.40-3.87$ & 0.001 \\
\hline Instillation & Pirarubicin $v s$. none & 0.036 & 2.07 & $1.10-3.89$ & 0.024 \\
\hline
\end{tabular}

Statistically significant $p$-values are shown in bold.

this study, we compared the efficacy of a single pirarubicin instillation with a short retention time to TURBT alone in patients with low-risk NMIBC.

\section{Patients and Methods}

Patient population. One hundred and sixty-five patients with lowrisk NMIBC who were treated at Shiga University of Medical Science Hospital and affiliated hospitals between 2006 and 2015 were included in this study. The histological diagnosis of all patients was urothelial carcinoma. Low risk was defined according to the Japanese Urological Association clinical practice guidelines for bladder cancer (3), which includes a single lesion, primary lesion, size $<3 \mathrm{~cm}$, Ta, low grade (12) and no concurrent carcinoma in situ. This definition corresponds to a recurrence score of 0 to 1 in the European Organization for Research and Treatment of Cancer risk table (4).
Patient management. TURBT was performed under white light, and no photodynamic diagnosis technique or narrow band imaging was applied in this study. IPSI was not given when bladder perforation was suspected. After complete TURBT, an immediate single dose of pirarubicin ( $30 \mathrm{mg}$ in $30 \mathrm{ml}$ of normal saline) was administered through a Foley catheter in the operating room, and the catheter was clamped. Intravesical pirarubicin retention time was $15 \mathrm{~min}$. Follow-up cystoscopy and urine cytology were performed at 3month intervals for the first year, biannually up to 5 years, and annually thereafter. Abdominal imaging was performed depending on the situation. Recurrence was defined as the presence of macroscopic tumor at cystoscopy and a subsequent diagnosis of urothelial carcinoma with TURBT.

Clinical data acquisition. All clinical and pathological data were collected from medical records. This was a retrospective observational study and was approved by Shiga University of Medical Science Hospital (approval number 30-007). 


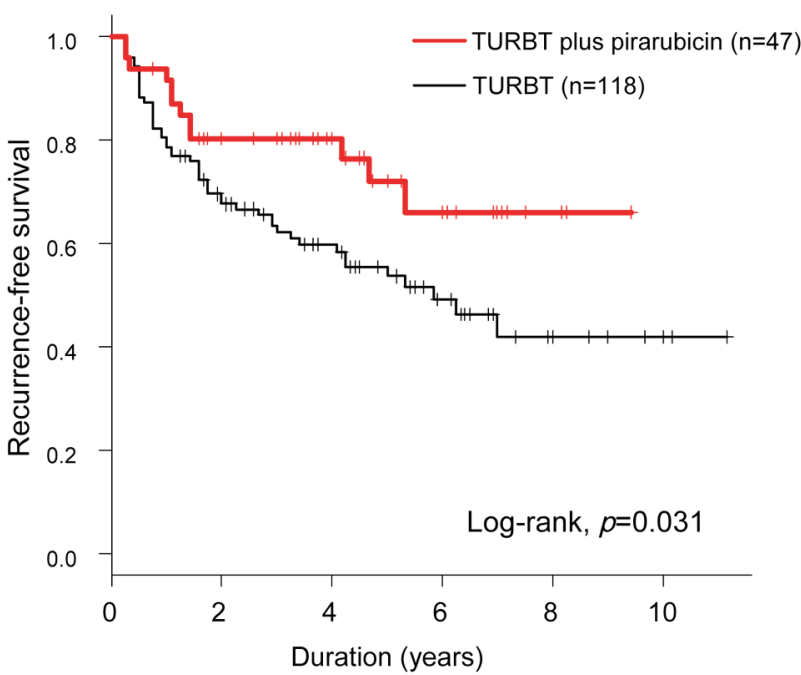

Figure 1. Kaplan-Meier analysis of recurrence-free survival in the groups treated with transurethral resection of bladder tumor (TURBT) alone and with pirarubicin.

In vitro study. In order to obtain supportive evidence of the effectiveness of short pirarubicin instillation therapy, cellular experiments were performed as described below. Three bladder cancer cell lines, UMUC-3, T24 (American Type Culture Collection, Rockville, MD, USA) and RT112 (European Collection of Authenticated Cell Cultures, Porton Down, UK) were used. Cell culture was performed using Dulbecco's modified Eagle's medium with $10 \%$ fetal bovine serum and penicillin $(100 \mathrm{IU} / \mathrm{ml})$ streptomycin $(100 \mu \mathrm{g} / \mathrm{ml})$ solution (Nacalai Tesque, Kyoto, Japan) under standard culture conditions, at $37^{\circ} \mathrm{C}$ with $5 \% \mathrm{CO}_{2}$. Cell viability measurement was performed with the Cell Counting Kit-8 assay solution (Dojindo, Kumamoto, Japan) according to the manufacturer's instructions. Cells ( $3 \times 10^{3}$ cells, $100 \mu \mathrm{l} /$ well) were seeded in 96 -well plates for $24 \mathrm{~h}$, and different concentrations of pirarubicin or MMC were applied for 15 and $60 \mathrm{~min}$. The cells were then thoroughly washed twice in phosphate-buffered saline and were cultured in normal medium. Three days after anticancer drug treatment, WST- 8 assay was performed and $50 \%$ inhibitory concentration $\left(\mathrm{IC}_{50}\right)$ values were calculated.

Statistical analysis. Recurrence-free survival (RFS) was estimated using Kaplan-Meier curve and statistically analyzed (log-rank test) using EZR software (13). Hazard ratios for intravesical recurrence were calculated using the Cox hazards model. Statistical analyses of clinical data were performed using Mann-Whitney $U$-test and chisquare test. In the analyses of cellular experiments, Student's $t$-test was used. A value of $p<0.05$ was considered statistically significant.

\section{Results}

Clinical study. Characteristics of patients in the pirarubicintreated and TURBT-only groups are shown in Table I, and clinical characteristics were not statistically different between the two groups. One- and five-year RFS were 91\% and $72 \%$ for the pirarubicin-treated, and $79 \%$ and $54 \%$ for

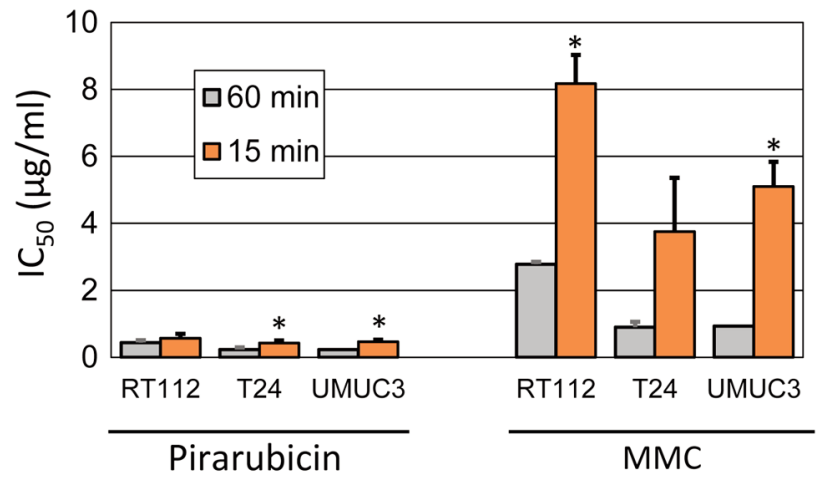

Figure 2. Half-maximal inhibitory concentration $\left(I_{50}\right)$ values for pirarubicin and mitomycin $C(M M C)$ against three bladder cancer cell lines. Data are means of triplicate experiments. Bars indicate standard deviation. *Significantly different at $p<0.05$.

the TURBT-only group (Figure 1). Pirarubicin IPSI was associated with a higher RFS than TURBT alone (log-rank, $p=0.031$ ). To clarify poor prognostic factors, we performed Cox hazards analysis (Table II). Larger tumor size $(>10 \mathrm{~mm})$ and lack of intravesical instillation therapy were revealed as significant risk factors for intravesical recurrence. Neither progression to MIBC nor adverse events regarding intravesical therapy were observed in any of the patients.

Cellular study. Figure 2 shows the $\mathrm{IC}_{50}$ values obtained following 15-min and 60-min exposure of UMUC3, T24 and RT112 cells to each of the drugs. In all three cell lines, pirarubicin exerted a strong antiproliferative effect at very low concentrations. Even when the exposure time was reduced from 60 to $15 \mathrm{~min}$, this tendency was consistent.

\section{Discussion}

Postoperative instillation has an important role in the prevention of intravesical recurrence of low- or intermediaterisk NMIBC (6). Especially in low-risk NMIBC, IPSI is strongly recommended (3-5). Although IPSI is listed as the standard of care in many clinical guidelines, its actual use in clinical practice varies widely across the globe $(7,14,15)$. For example, clinical practice surveys have revealed that 66 and $28 \%$ of urologists in the U.S. and Europe, respectively, have never used IPSI. The low rate of adoption may be due to several factors, including individual surgeon's decisions or preferences, the surgeon's workload and educational degree, the level of nursing care, and the degree to which pharmacies are prepared $(14,15)$. However, as we have shown, TURBT alone is an inadequate treatment because it is associated with a significantly higher recurrence rate than is IPSI. 
MMC, epirubicin and pirarubicin are all thought to be beneficial treatments (4). A recent randomized study of IPSI with MMC and pirarubicin showed no statistically difference in RFS (16). Most previous instillation studies of pirarubicin used a retention time of $1 \mathrm{~h}(8,17)$. Pirarubicin was shown in the 1980 s to be rapidly taken up by cultured tumor cells (10). Several clinical trials evaluating short-duration (retention time 5-15 min) instillation using pirarubicin have been carried out $(11,18)$. Histological analysis has revealed that installation with pirarubicin for $5 \mathrm{~min}$ before transurethral biopsy of bladder tumors is sufficient for adequate uptake of the drug (11). Han et al. reported "pirarubicin endoscopy", in which they exploited the autofluorescence of pirarubicin to measure its uptake via blue light cystoscopy (18). After 15-min instillation of pirarubicin, bright red fluorescence in bladder tumors demonstrated the rapid uptake of the drug. According to these findings, we performed pirarubicin chemotherapy with a short instillation time (15 $\mathrm{min})$ in the setting of IPSI for lowrisk NMIBC. Our in vitro study using bladder cancer cell lines also supports these findings. Based on our clinical and experimental data, short-retention IPSI of pirarubicin is effective in preventing intravesical recurrence of low-risk NMIBC. In part, the complexity of postoperative care following IPSI explains its low adoption rate in clinical practice (15). A reduced retention time might benefit surgeons and other medical staff. Moreover, the duration of observation required in the recovery room could probably be shortened. Recently, intravesical gemcitabine was applied to IPSI (19-21). Although safety and tolerability of gemcitabine was reported in the IPSI setting, a longer drug dwell time ( $>1 \mathrm{~h})$ seems to be needed to obtain an antitumor effect $(20,21)$.

Generally, immediate postoperative instillation induced minimal adverse events, which typically comprised chemical cystitis and skin irritation (22). However, there have been some case reports of severe adverse events due to extravasation. Oddens et al. reported three cases of severe complications caused by extravasation of epirubicin (23). One of these cases presented with intraperitoneal extravasation, and died from multiple organ failure associated with paralytic ileus. Filson et al. reported that major complications (Clavien-Dindo classification grade 3 or more) were observed in $5.2 \%$ of patients treated with perioperative $\mathrm{MMC}$, with one patient needing radical cystectomy (24). Therefore, all clinical guidelines strongly recommend avoiding immediate postoperative instillation in any cases where bladder perforation is suspected (3-5). However, small non-visualized perforations of the bladder may occur even when TURBT is performed carefully. For example, Balbay et al. reported extravasation in 58\% of patients after TURBT, even when surgeons did not suspect bladder perforation in these patients (25). Considering the above findings, shortening the intravesical retention time might be a reasonable strategy for avoiding severe extravasation of chemotherapeutic drugs. To test this hypothesis, larger clinical studies are essential.

There are several limitations of the present study. Firstly, our cohort size was too small to derive generalizable conclusions from the findings, and the follow-up period was relatively short, especially for the MMC group. Secondly, our analysis was retrospective in nature. A prospective randomized control study is needed. Thirdly, the use of intravesical pirarubicin is not covered by health insurance in many Western countries. Despite these limitations, our results provide new insight into good practice for prevention of intravesical recurrence in patients with low-risk NMIBC. Validation of this treatment strategy will depend upon future follow-up studies.

In conclusion, almost half of patients with low-risk NMIBC encountered intravesical recurrence with TURBT alone. Short retention IPSI using pirarubicin can prevent intravesical recurrence in patients with low-risk NMIBC.

\section{Conflicts of Interest}

The Authors have no conflicts of interest directly relevant to the content of this article.

\section{Authors' Contributions}

S. Kageyama, K.K., and S. Kubota conceptualized and designed the study. T.O., Y.A., H.S., Z.N., Y.S., K.T., and C. J. K. involved in acquisition of data. C. J. K., T.C., T.Y., and A.K. involved in analysis and interpretation of data. All the Authors were involved in manuscript for all aspects of the work.

\section{Acknowledgements}

The Authors gratefully thank Ms. Hiroko Kita and Ms. Masami Katsurai for their assistance in laboratory experiments.

\section{References}

1 GLOBCAN 2018. Cancer fact sheet: Bladder. Available at: http://gco.iarc.fr/today/data/factsheets/cancers/30-Bladder-factsheet.pdf [Last accessed on November 14, 2020]

2 Kamat AM, Hahn NM, Efstathiou JA, Lerner SP, Malmström PU, Choi W, Guo CC, Lotan Y and Kassouf W: Bladder cancer. Lancet 388(10061): 2796-2810, 2016. PMID: 27345655. DOI: 10.1016/S0140-6736(16)30512-8

3 Kubota Y, Nakaigawa N, the Committee for Establishment of the Clinical Practice Guideline for the Management of Bladder Cancer and the Japanese Urological Association: Essential content of evidence-based clinical practice guidelines for bladder cancer: The Japanese Urological Association 2015 update. Int J Urol 23(8): 640-645, 2016. PMID: 27374472. DOI: 10.1111/iju.13141

4 EAU Guideline on Non-muscle-invasive Bladder Cancer (TaT1 and CIS) 2019. Available at: https://uroweb.org/guideline/nonmuscle-invasive-bladder-cancer/ [Last accessed on November 14, 2020]

5 AUA/SUO guideline. Diagnosis and Treatment of Non-muscleinvasive Bladder Cancer: AUA/SUO joint guideline (2016). 
Available at: https://www.auanet.org/guidelines/bladder-cancernon-muscle-invasive-guideline [Last accessed on November 14, 2020]

6 Sylvester RJ, Oosterlinck W, Holmang S, Sydes MR, Birtle A, Gudjonsson S, De Nunzio C, Okamura K, Kaasinen E, Solsona E, Ali-El-Dein B, Tatar CA, Inman BA, N'Dow J, Oddens JR and Babjuk M: Systematic review and individual patient data meta-analysis of randomized trials comparing a single immediate instillation of chemotherapy after transurethral resection with transurethral resection alone in patients with stage pTa-pT1 urothelial carcinoma of the bladder: Which patients benefit from the instillation? Eur Urol 69(2): 231-244, 2016. PMID: 26091833. DOI: $10.1016 /$ j.eururo.2015.05.050

7 Burks FN, Liu AB, Suh RS, Schuster TG, Bradford T, Moylan DA, Knapp PM, Murtagh DS, Dunn RL, Montie JE and Miller DC: Understanding the use of immediate intravesical chemotherapy for patients with bladder cancer. J Urol 188(6): 2108-2113, 2012. PMID: 23083865. DOI: 10.1016/j.juro.2012.08.044

8 Okamura K, Ono Y, Kinukawa T, Matsuura O, Yamada S, Ando T, Fukatsu T, Ohno Y, Ohshima S; Nagoya University Urological Oncology Group: Randomized study of single early instillation of (2"R)-4'-O-tetrahydropyranyl-doxorubicin for a single superficial bladder carcinoma. Cancer 94(9): 2363-2368, 2002. PMID: 12015761. DOI: 10.1002/cncr.10496

9 Sylvester RJ, Oosterlinck W and van der Meijden AP: A single immediate postoperative instillation of chemotherapy decreases the risk of recurrence in patients with stage Ta T1 bladder cancer: a meta-analysis of published results of randomized clinical trials. J Urol 171(6): 2186-2190, 2004. PMID: 15126782. DOI: 10.1097/01.ju.0000125486.92260.b2

10 Kunimoto S, Miura K, Takahashi Y, Takeuchi T and Umezawa $\mathrm{H}$ : Rapid uptake by cultured tumor cells and intracellular behavior of 4'-O-tetrahydropyranyladriamycin. J Antibiot 36(3): 312-317, 1983. PMID: 6833151. DOI: 10.7164/antibiotics. 36.312

11 Uchikoba T, Horiuchi K, Oka F, Sato M, Tsuboi N, Ohaki Y and Nishimura T: Diagnosing the location of carcinoma in situ (CIS) of the urinary bladder using pirarubicin hydrochloride. Urol Int 74(3): 235-239, 2005. PMID: 15812210. DOI: 10.1159/000 083555

12 Eble JN, Sauter G, Epstein JE and Sesterhenn IA (eds.): Tumours of the Urinary System and Male Genital Organs (World Health Organization Classification of Tumours). Lyon, IARC Press, 2004.

13 Kanda Y: Investigation of the freely available easy-to-use software 'EZR' for medical statistics. Bone Marrow Transplant 48(3): 452-458, 2013. PMID: 23208313. DOI: 10.1038/bmt. 2012.244

14 Cookson MS, Chang SS, Oefelein MG, Gallagher JR, Schwartz B and Heap K: National practice patterns for immediate postoperative instillation of chemotherapy in nonmuscle-invasive bladder cancer. J Urol 187(5): 1571-1576, 2012. PMID: 22425105. DOI: 10.1016/j.juro.2011.12.056

15 Palou-Redorta J, Rouprêt M, Gallagher JR, Heap K, Corbell C and Schwartz B: The use of immediate postoperative instillations of intravesical chemotherapy after TURBT of NMIBC among European countries. World J Urol 32(2): 525-530, 2014. PMID: 23907663. DOI: $10.1007 / \mathrm{s} 00345-013-1142-\mathrm{z}$

16 Yamamoto S, Kageyama Y, Fujii Y, Aizawa T, Urakami S and Fukui I: Randomized study of postoperative single intravesical instillation with pirarubicin and mitomycin $\mathrm{C}$ for low-risk bladder cancer. Anticancer Res 40(9): 5295-5299, 2020. PMID: 32878820. DOI: 10.21873/anticanres.14535

17 Tanimoto R, Saika T, Ebara S, Kobayashi Y, Nasu R, Yamada D, Takamoto H, Miyaji Y, Nasu Y, Tsushima T and Kumon H: Prospective randomized controlled trial of postoperative early intravesical chemotherapy with pirarubicin (THP) for solitary non-muscle-invasive bladder cancer comparing single and twotime instillation. World J Urol 36(6): 889-895, 2018. PMID: 29387931. DOI: 10.1007/s00345-018-2196-8

18 Han J, Lin T, Xu K, Jiang C, Huang H, Yin X, Xie W, Yao Y, Zhang $\mathrm{C}$ and Huang $\mathrm{J}$ : Improved detection of nonmuscleinvasive urothelial carcinoma of the bladder using pirarubicin endoscopy: a prospective, single-center preliminary study. J Endourol 24(11): 1801-1806, 2010. PMID: 20932082. DOI: 10.1089/end.2009.0680

19 NCCN clinical practice guidelines in oncology. Bladder Cancer. Ver. 3. 2020. Available at: https://www.nccn.org/professionals/ physician_gls/pdf/bladder.pdf [Last accessed on November 14, 2020]

20 Böhle A, Leyh H, Frei C, Kühn M, Tschada R, Pottek T, Wagner W, Knispel HH, von Pokrzywnitzki W, Zorlu F, Helsberg K, Lübben B, Soldatenkova V, Stoffregen C, Büttner H; S274 Study Group: Single postoperative instillation of gemcitabine in patients with non-muscle-invasive transitional cell carcinoma of the bladder: a randomised, double-blind, placebo-controlled phase III multicentre study. Eur Urol 56(3): 495-503, 2009. PMID: 19560257. DOI: 10.1016/j.eururo.2009.06.010

21 Messing EM, Tangen CM, Lerner SP, Sahasrabudhe DM, Koppie TM, Wood DP Jr, Mack PC, Svatek RS, Evans CP, Hafez KS, Culkin DJ, Brand TC, Karsh LI, Holzbeierlein JM, Wilson SS, Wu G, Plets M, Vogelzang NJ and Thompson IM Jr: Effect of intravesical instillation of gemcitabine $v s$. saline immediately following resection of suspected low-grade nonmuscle-invasive bladder cancer on tumor recurrence: SWOG S0337 randomized clinical trial. JAMA 319(18): 1880-1888, 2018. PMID: 29801011. DOI: 10.1001/jama.2018.4657

22 Zamboni S, Baumeister P, Mattei A, Mordasini L, Antonelli A, Simeone $\mathrm{C}$, and Moschini M: Single postoperative instillation for non-muscle-invasive bladder cancer: are there still any indication? Transl. Androl Urol 8(1): 76-84, 2019. PMID: 30976571. DOI: $10.21037 /$ tau.2018.08.20

23 Oddens JR, van der Meijden AP and Sylvester R: One immediate postoperative instillation of chemotherapy in low risk $\mathrm{Ta}, \mathrm{T} 1$ bladder cancer patients. Is it always safe? Eur Urol 46(3): 336-338, 2004. PMID: 15306104. DOI: 10.1016/j.eururo.2004. 05.003

24 Filson CP, Montgomery JS, Dailey SM, Crossley HS, Lentz H, Tallman CT, He C and Weizer AZ: Complications associated with single-dose, perioperative mitomycin-C for patients undergoing bladder tumor resection. Urol Oncol 32(1): 40.e1-8, 2014. PMID: 23787296. DOI: 10.1016/j.urolonc.2013.04.006

25 Balbay MD, Cimentepe E, Unsal A, Bayrak O, Koç A and Akbulut Z: The actual incidence of bladder perforation following transurethral bladder surgery. J Urol 174(6): 2260-2262, 2005. PMID: 16280794. DOI: 10.1097/01.ju.0000181811.61199.35

Received November 16, 2020

Revised December 7, 2020

Accepted December 8, 2020 